Theories \& Applications, the International Edition

Printed Version : (ISSN 2090-5262)

Online Version : (ISSN 2090-5270)

November 2014, Volume 4, No. 3 Pages (58 - 64)

\title{
The Effect of Maximum Physical Effort on the Level of Free Radicals after Extra Antioxidants Supplementation.
}

\section{Prof.Hamdy Abdou Asem}

Professor, Department of Health Science Sports Sadat City University, Egypt.

\section{Dr. Abd El Halem Youssef Abd El Aleam}

Assistant Professor, Department of Health Science Sports Sadat city University, Egypt.

\section{Dr. Kalled Hessin Ezaat}

Assistant Professor, Department of Sport Training College of Physical Education, Tanta University, Egypt.

\begin{abstract}
The present study was designed to investigate the effects of maximum physical effort on free radicals after Extra antioxidan ts supplementation vitamin C (ascorbic acid; 1,000 mg/day), and vitamin E (tocopherol; $800 \mathrm{mg} /$ day) orally for 4 week. on the time course of MaloneAldehde, , Lactic acid, VO2 max and Exercise duration after maximum physical effort.
\end{abstract}

The question that arises is, how effectively can athletes defend against the increased free radicals resulting from exercise? Do athletes need to take extra antioxidants supplementation?

\section{Experimental procedure:}

Objective of the research :

The current research aims to identify the impact of the extra intake of antioxidants on:

1. The level of production Malondialdehyde (UmolL)

2. The level of lactic acid (MmolN)

3. Maximum oxygen consumption VO2 max ml_kg_1_min

4. Exercise duration s (second)

Sixteen players from the youth handball Giza Club after explanation of all experimental procedures and signed the written informed consent were divided into two groups each of them (8) players Participants were placed into one of two groups were given the first group vitamin $C$ (ascorbic acid; 1,000 $\mathrm{mg} /$ day), and vitamin $E$ (tocopherol; $800 \mathrm{mg} /$ day) orally for 4 weak. No subject was a smoker, used oral contraceptives, anti-inflammatory drugs, or dietary supplements (i.e., antioxidants)

The subjects performed a VO2 max cycle ergometer exercise test. The test workload was started at $50 \mathrm{~W}$ and increased by 50 $W$ every 2 min while the subjects maintained a pedal frequency of 50 revolutions/ min. The exercise tests lasted 8-12 min and terminated when the subjects indicated exhaustion or when the heart rate reached to maximal heart rate calculated as 220 - age.

Within the limits of the research sample and through the research results the following are shown:

1. The test workload (maximum physical effort) Cause in increased production of free radicals, based on the (Malondialdehyde)

2. Both of Vitamin $(C)$ and $(E)$ had a positive impact on MaloneAldehde, Lactic acid, VO2 max and Exercise duration in reducing the productivity of free radicals.

3. Vitamin ( $E)$ was more effective in increasing the performance time during the test workload (maximum physical effort)

4. Very important for athletes who are exposed repeatedly to high-intensity loads take a extra Antioxidants supplementation.

Introduction

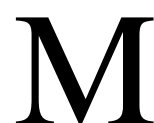

ost of the oxygen consumed in the mitochondria during energy metabolism combines with

hydrogen to produce water. Normally, about $2 \%$ to $5 \%$ of oxygen forms the oxygen-containing free radicals superoxide (O2_), hydrogen peroxide ( $\mathrm{H} 2 \mathrm{O} 2)$, and hydroxyl $\left(\mathrm{OH} \_\right)$from electron "leakage" along the electron transport chain A free radical represents a chemically reactive molecule or molecular fragment with at least one unpaired electron in its outer orbital or valence shell. A buildup of free radicals increases the potential for cellular damage or oxidative stress to biologically important substances Increased Metabolism during Exercise and Free Radical Production. Oxygen radicals exhibit strong affinity for the polyunsaturated fatty acids in the lipid baitlayer of cell membranes. During oxidative stress, deterioration occurs in the plasma membrane's fatty acids. Membrane damage occurs through a series of chain reactions termed lipid peroxidation. These reactions, which incorporate oxygen into lipids, increase the vulnerability of the cell and its 
constituents and a general decline in central nervous system and immune function.(28)

Acute exercise is involved in several ways. During the aerobic production of ATP, single electrons leak from electron transport in Stage IV in the mitochondria .

The higher the rate of metabolism (as in moving from rest to submaximal to maximal exercise), the more free radicals are produced. Possibly as much as $4-5 \%$ of the oxygen consumed is converted to free radicals. Anaerobic energy production provides an abundance of hydrogen ions that can react with an oxygen-free radical to form a ROS, such as hydrogen peroxide, $\mathrm{H} 2 \mathrm{O} 2$. Hypoxia leads to freeing of metals $(\mathrm{Fe}, \mathrm{Cu}$, and $\mathrm{Mg}$ ) that are needed to catalyze free radical production. Exercise-induced hyperthermia may trigger free radical proliferation. Any damage to muscle fibers leads to increased immune response.

Despite the increased production of free radicals resulting from exercise, it is unlikely that exercise results in substantial damages to a normal healthy individual.

All individuals, but especially those doing high-intensity training, should make sure that their diets contain large amounts of antioxidant- beneficial and antioxidants Supplementation. (24)

Many studies have shown that unaccustomed exercise, especially eccentric exercise, results in damage to muscle structure and soreness. It is recognized that free radicals, highly reactive chemical species, may be involved in the etiology of this damage to muscle membrane and resultant soreness. Quenching the effects of free radicals may reduce the muscle damage and pain, and thereby allow for greater training. Furthermore, free radical production is likely to be enhanced as a result of increases in oxygen consumption (which occurs during exercise) since they are produced through electron escape from the electron transport chain.(13)

Free radicals are generated from normal oxidative processes in the body and can damage DNA and RNA and inactivate enzymes and other proteins. Free radicals also facilitate oxidation of fatty acids in cell membranes, producing destructive chain reactions that cause cell damage and cell death. Excess levels of free radicals in plasma and the arterial wall increase low-density lipoprotein (LDL) oxidation, leading to cytotoxicity and enhanced plaque formation. (1)

Aerobic organisms would not survive without mechanisms that counteract the detrimental effects of free radicals. The system includes the fat-soluble antioxidants such as vitamin $\mathrm{E}$ and beta-carotene (a vitamin A precursor); the major water-soluble antioxidant, vitamin $\mathrm{C}$; antioxidant enzymes such as superoxide dismutase (SOD), catalase (CAT), and selenium-dependent glutathione peroxidase (GPX); and lowmolecular-weight compounds such as glutathione.(2) These components preserve homeostasis during most normal cell function and mild oxidative stress. When free-radical production is excessive, however, or when the antioxidant system is overwhelmed, such as during nutritional deficiencies or exhaustive exercise, such imbalances may favor an "oxidative stress" environment.

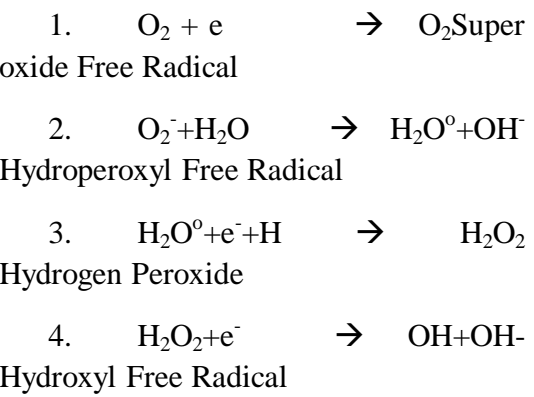

Although both enzymes share the primary function of reducing hydrogen peroxide, their substrate specificity and cellular location are different. CAT is found primarily in peroxisomes, organelles involved in no mitochondrial oxidation of fatty acids and amino acids, and they generate hydrogen peroxide. GPX, a mitochondrial enzyme, uses glutathione, a low-molecular-weight thiol, to reduce hydrogen to water and organic peroxides to alcohol. Thus, GPX plays an important role in inhibiting lipid peroxidation and preventing damage to DNA and RNA. (2)

A second pathway is in organs, such as the liver, kidneys, and gut, that experience a hypoxic environment with bloodflow redistribution to the working muscles. This relative ischemia-reperfusion of the splanchnic region may trigger activation of xanthine oxidase, a membrane-bound enzyme that produces both superoxide and hydrogen peroxide. Finally, neutrophil and macrophage involvement in inflammation and repair may be a potent source of freeradical production.(3)

Given that high-intensity exercise can increase free-radical production, antioxidant supplements may offer benefit during prolonged aerobic activity.

\section{The research problem and its importance:}

Conflicting data exist; the preponderance of available information suggests that physical exercise promotes an increase in free-radicals. We have Few studies Interested measured exercise-induced free radicals directly, the measurement of lipid peroxidation as the principal indicator of exercise-induced free radicals. It should be noted that free radicals can also alter and inactivate enzyme complexes, damage DNA and RNA, and promote mutations effects. It is not presently known if taken extra antioxidant supplementation will affect these positive aspects of free radicals during prolonged exercise activity

The question that arises is, how effectively can athletes defend against the increased free radicals resulting from exercise? Do athletes need to take extra antioxidants supplementation?

\section{Experimental procedure:}

Objective of the research: 
The current research aims to identify the impact of the extra intake of antioxidants on:

1. The level of production Malondialdehyde (Umol $\backslash \mathrm{L})$

2. The level of lactic acid (MmollL)

3. Maximum oxygen consumption $\mathrm{VO}_{2} \quad \max$ ml_kg_1_min

4. Exercise duration s (second)

\section{The Methodology}

The Experimental method which is appropriate to the nature of the study

\section{Experimental Protocol:}

All subjects performed the exercise test (maximum physical effort) at the initial phase of their participation in the study. After this first test, they were asked to take group vitamin C (ascorbic acid; $1,000 \mathrm{mg} /$ day), and group vitamin $\mathrm{E}$ (tocopherol; $800 \mathrm{mg} /$ day) All subjects then performed another (maximum physical effort) test as described above at the end of this 4 week period

\section{Materials and Methods}

Subjects: Sixteen players from the Junior handball Giza Club after explanation of all experimental procedures and signed the written informed consent were separated into two groups, were tested. The first group (GC) was composed of 8 players (age $18.6 \pm 0.7 \mathrm{y}$ ). The second group (GE) consisted of 8 players (age $18.7 \pm 0.8$ y) were given the group (GC) vitamin C (ascorbic acid; $1,000 \mathrm{mg} /$ day), and group (GE) vitamin E (tocopherol; $800 \mathrm{mg} /$ day) orally for 4 weak. No subject was a smoker, used oral contraceptives, anti-inflammatory drugs, or dietary supplements (i.e., antioxidants).

Exercise Model: The subjects performed a $\mathrm{VO}_{2}$ max cycle ergometer exercise test. The test workload was started at 50 $\mathrm{W}$ and increased by $50 \mathrm{~W}$ every $2 \mathrm{~min}$ while the subjects maintained a pedal frequency of 50 revolutions/ min. The exercise tests lasted_8-12 $\mathrm{min}$ and terminated when the subjects indicated exhaustion or when the heart rate reached to maximal heart rate calculated as( 220 - age) .

Table 1:

Mean comparison of Resting Malondialdehyde and Lactic acid in Vitamin (E) and Vitamin (C) groups $(n=8)$

\begin{tabular}{|c|c|c|c|c|c|c|}
\hline Variables & Mean & Std deviation & skewness & kurtosis & $\begin{array}{c}\text { Mean } \\
\text { Difference }\end{array}$ & $\mathrm{T}$ \\
\hline Malondialdehyde Rest ( Umol\L) GC & .82 & $.0707 \pm$ & .43 & -1.40 & \multirow{2}{*}{0.067} & \multirow{2}{*}{-3.70} \\
\hline Malondialdehyde Rest ( UmollL) GE & .89 & $.0659 \pm$ & .86 & .41 & & \\
\hline Lactic acid Rest (MmollL) GC & .81 & $.0390 \pm$ & -.22 & -1.24 & \multirow{2}{*}{0.02} & \multirow{2}{*}{-0.49} \\
\hline Lactic acid Rest (MmollL)GE & .83 & $.0479 \pm$ & 1.19 & 2.19 & & \\
\hline Heart rate GC & 65.5 & 1.60 & 0.00 & -.31 & \multirow{2}{*}{0.7} & \multirow{2}{*}{0.615} \\
\hline Heart rate GE & 64.8 & 2.03 & 0.22 & -.88 & & \\
\hline
\end{tabular}

Values are means _ SE, Skewness and Kurtosis No Significant difference from the value between two group at rest

Table 2:

Malondialdehyde, Lactic acid, VO2 max and Exercise duration In Vitamin (C) And Vitamin (E) groups after test workload before Antioxidants supplementation.

$(\mathbf{n}=8)$

\begin{tabular}{|c|c|c|c|c|c|c|}
\hline Variables & Mean & $\begin{array}{c}\text { Std } \\
\text { deviation }\end{array}$ & skewness & kurtosis & $\begin{array}{c}\text { Mean } \\
\text { Difference }\end{array}$ & $\mathrm{T}$ \\
\hline Malondialdehyde ( UmollL) GC & 1.82 & $.13 \pm$ & .310 & -.99 & \multirow{2}{*}{0.058} & \multirow{2}{*}{.368} \\
\hline Malondialdehyde ( UmollL) GE & 1.76 & $-.28 \pm$ & .067 & 1.76 & & \\
\hline Lactic acid $(\mathrm{Mmol} \backslash \mathrm{L}) \mathrm{GC}$ & 4.07 & $1.05 \pm$ & .756 & -.24 & \multirow{2}{*}{0.088} & \multirow{2}{*}{-.658} \\
\hline Lactic acid (MmolLL) GE & 3.99 & $.166 \pm$ & .131 & -1.58 & & \\
\hline $\mathrm{VO}_{2}$ max ml_kg_1_min $\mathrm{GC}$ & 56.88 & $.851 \pm$ & -.460 & -.44 & \multirow{2}{*}{0.13} & \multirow{2}{*}{2.577} \\
\hline $\mathrm{VO}_{2}$ max ml_kg_1_min GE & 57.01 & $.182 \pm$ & .466 & .042 & & \\
\hline Exercise duration $\mathrm{s}$ G C & 574.75 & $13.96 \pm$ & -.341 & 1.77 & \multirow{2}{*}{-0.25} & \multirow{2}{*}{-.081} \\
\hline Exercise duration $\mathrm{s}$ GE & 575.00 & $13.03 \pm$ & -.614 & -1.52 & & \\
\hline
\end{tabular}

Values are means _ SE. Significant difference from the value after test workload before Antioxidants supplementation.

\section{Data collection tools:}

1. Fitmate device for test (Resting metabolism, Cardiorspiratory fitness $\left.\left(\mathrm{VO}_{2 \max }\right)\right)$ 
2. Heart Rate Monitor (Polar) RS800CX SD

3. Lactic Accupsports Test using standard tapes Strip Test

4. Autoclix $\backslash 25$ to take a blood sample to measure the level of lactic.

5. Bicycle ERGO BIKE

6. Spectral analysis Spectrophotometer

7. Medical Syringes

8. Centrifuge

9. Pipettes
10. Water path
11. Kites
12. Metronome
13. Cotton wool
14. Numbered tubes and sterilized bottles.

\section{Presenting and discussing the results:}

All subjects in the vitamin $\mathrm{C}$ and vitamin $\mathrm{E}$ groups completed the exercise protocol without problems, and reached to maximal heart rate calculated as $(220-$ age $)$

Table 3 :

Mean comparison of Malondialdehyde and Lactic acid In

Vitamin (C) And Vitamin (E) groups test workload before Antioxidants supplementation. (n=8)

\begin{tabular}{|c|c|c|c|c|c|c|}
\hline \multirow[b]{2}{*}{ Variables } & \multicolumn{2}{|c|}{ Rest before workload } & \multicolumn{2}{|c|}{ After workload } & \multirow[b]{2}{*}{ Difference } & \multirow[b]{2}{*}{$\mathrm{T}$} \\
\hline & Mean & $\begin{array}{c}\text { Std } \\
\text { deviation }\end{array}$ & Mean & $\begin{array}{c}\text { Std } \\
\text { deviation }\end{array}$ & & \\
\hline Malondialdehyde ( UmollL) GC & .071 & $.83 \pm$ & 1.82 & $.13 \pm$ & 1.11 & $*-19.741$ \\
\hline Malondialdehyde ( UmollL) GE & .066 & $.97 \pm$ & 1.76 & $.068 \pm$ & 1.70 & $*-24.914$ \\
\hline Lactic acid $(\mathrm{Mmol} / \mathrm{L}) \mathrm{GC}$ & .87 & $.039 \pm$ & 4.08 & $.17 \pm$ & -3.99 & $* 59.753$ \\
\hline Lactic acid $(\mathrm{Mmol} \backslash \mathrm{L}) \mathrm{GE}$ & .88 & $.048 \pm$ & 3.99 & $.18 \pm$ & -3.11 & $*-43.770$ \\
\hline
\end{tabular}

Values are means _ SE. Significant difference from the value before Antioxidants supplementation $* P_{-} 0.05$;

Table 4

Mean comparison of Malondialdehyde, Lactic acid, VO2maxand Exercise duration In Vitamin (C) and Vitamin (E) groups test workload after Antioxidants supplementation. $\quad(n=8)$

\begin{tabular}{|c|c|c|c|c|c|c|}
\hline \multirow{2}{*}{ Variables } & \multicolumn{2}{|c|}{$\begin{array}{c}\text { Before Antioxidants } \\
\text { supplementation. }\end{array}$} & \multicolumn{2}{|c|}{$\begin{array}{l}\text { After Antioxidants } \\
\text { supplementation. }\end{array}$} & \multirow{2}{*}{ Difference } & \multirow{2}{*}{$\mathrm{T}$} \\
\hline & Mean & $\begin{array}{c}\text { Std } \\
\text { deviation }\end{array}$ & Mean & $\begin{array}{c}\text { Std } \\
\text { deviation }\end{array}$ & & \\
\hline Malondialdehyde $\quad($ Umol $\backslash$ L) GC & 1.82 & $.13 \pm$ & .70 & $.25 \pm$ & 1.12 & $* 10.199$ \\
\hline Malondialdehyde & 1.76 & $.068 \pm$ & .81 & $.046 \pm$ & 0.95 & $* 29.790$ \\
\hline Lactic acid $(\mathrm{Mmol} L) \mathrm{GC}$ & 4.08 & $.167 \pm$ & 3.76 & $.32 \pm$ & 0.32 & 2.796 \\
\hline Lactic acid (MmollL) GE & 3.99 & $.18 \pm$ & 3.76 & $.17 \pm$ & 0.23 & $* 7.806$ \\
\hline $\mathrm{VO}_{2 \max }$ ml_kg_1_min GC & 56.89 & $1.06 \pm$ & 57.57 & $.85 \pm$ & 0.69 & $*-5.373$ \\
\hline $\mathrm{VO}_{2 \max }$ ml_kg_1_min GE & 57.01 & $.855 \pm$ & 58.44 & $.50 \pm$ & 1.42 & $*_{-6.790}$ \\
\hline Exercise duration s G C & 574.75 & $13.96 \pm$ & 581.38 & $17.00 \pm$ & 6.62 & -.883 \\
\hline Exercise duration s G E & 575.00 & $13.03 \pm$ & 612.87 & $18.25 \pm$ & 37.87 & $*-4.953$ \\
\hline
\end{tabular}

Values are means_ SE. Significant difference from the value test workload before Antioxidants supplementation $* P$ _ 0.05 ;

Table 5

Mean comparison of Malondialdehyde, Lactic acid, VO2 max and Exercise duration In Vitamin (C) and Vitamin (E) groups after test workload with Antioxidants supplementation

$(\mathbf{n}=8)$

\begin{tabular}{|c|c|c|c|c|c|c|}
\hline \multirow[b]{2}{*}{ Variables } & \multicolumn{2}{|c|}{ Group vitamin $(\mathrm{C})$} & \multicolumn{2}{|c|}{ Group vitamin $(\mathrm{E})$} & \multirow[b]{2}{*}{ Difference } & \multirow[b]{2}{*}{$\mathrm{T}$} \\
\hline & Mean & $\begin{array}{c}\text { Std } \\
\text { deviation }\end{array}$ & Mean & $\begin{array}{c}\text { Std } \\
\text { deviation }\end{array}$ & & \\
\hline Malondialdehyde ( UmollL) & .70 & .25 & .81 & .047 & 0.11 & -1.103 \\
\hline Lactic acid (MmollL) & 3.76 & .32 & 3.76 & .17 & 1.25 & -.011 \\
\hline $\mathrm{VO}_{2 \max } \mathrm{ml} \_k g \_1 \_\min$ & 57.58 & 1.20 & 58.43 & .50 & 0.87 & -2.238 \\
\hline Exercise duration $\mathrm{s}$ & 581.37 & 17.00 & 612.88 & 18.25 & 31.41 & $*-5.694$ \\
\hline
\end{tabular}


Values are means _ SE. Significant difference from the value test workload after Antioxidants supplementation $* P{ }_{-} 0.05$;

\section{Discussion and Conclusion}

\section{In Tabel 3:}

Average production of free radicals through the level (Malonedialdehyde Umol $L$ L) \& (Lactic acid MmollL) for the two groups of study after test workload and before taken Vitamin (E) \&Vitamin(C) Antioxidants supplementation For the benefit of test workload.

We find that test workload (maximum physical effort) Cause in increased production of free radicals, based on the evidence standard (Malone dialdehyde) and this increase was statistically significant and the research team believes that the reasons for increasing free radicals Cause by loads high intensity to increase metabolism in the case of increasing intensity effort and which results in free radicals and the so-called fatty dioxide.

Which concluded that training high intensity increases the production of roots oxygen evidence tension oxidation and damage oxidized in large muscles where most oxygen consumer during the production of energy in mitochondria combines with hydrogen and be water proof Furthermore. There is so much oxygen between 2 to $5 \%$ are the roots of oxygen Shared such as super oxide Desmiotiz and hairdoboxcaid during the various steps in the ring moving which in turn leads to mass cell indicates to the release of the roots of oxygen during physical activity may be through tracks at least first track through the transfer of electrons within mitochondria and second track through the change in blood flow and nutrition oxygen to muscles structural resulting in what is known as tension oxidative stress (12) (16) (23).

\section{In Tabel 4:}

Significant difference from the value test workload after Extra Antioxidants supplementation $\left({ }^{*} P_{-} 0.05\right)$ in both groups Malonedialdehyde Umol $\backslash \mathrm{L}$, Lactic acid MmollL, $\mathrm{VO}_{2}$ max ml_kg_1_min and group Vitamin ( E ) Exercise duration s test workload after Extra Antioxidants supplementation The results indicate that Extra Antioxidants supplementation to improvement because, as we'll show the impact of each of Vitamin $\mathrm{C} \& \mathrm{E}$

Vitamin C. Less convincing is the evidence for vitamin C supplement-mediated protection against exercise-induced oxidative damage. One study (10) demonstrated that ascorbate is an effective antioxidant in human plasma. Improved musculoskeletal healing has been reported in patients who took 500 to $1,000 \mathrm{mg}$ of ascorbic acid two to four times a day.(11) A recent review(20) notes that although large doses of vitamin $\mathrm{C}$ are claimed to reduce fatigue and muscle damage, none of the studies examined specific oxidative stress markers, and therefore such largedose strategies must be interpreted with some caution. Furthermore, high doses of vitamin $\mathrm{C}$ in the presence of iron can have pro-oxidant effects. (21) Investigators have speculated that free radicals may play a role in muscle damage associated with eccentric muscle activity.(25)

Vitamin E This fat-soluble vitamin is incorporated into lipoproteins and cell membranes and prevents oxidation of polyunsaturated fatty-acids in cell membranes. Vitamin E also inhibits platelet activation and monocyte adhesion (8) and is the predominant antioxidant in LDL. (28) (23) the most active and most common commercial form of vitamin (E) is alpha-tocopherol.

Vitamin E: This vitamin is essential for normal cell function during exercise. Animals depleted of vitamin E demonstrate significant free-radical generation, lipid peroxidative damage, and a $40 \%$ decline in endurance capacity (7) thus implicating vitamin $\mathrm{E}$ in protecting against exercise-induced free-radical generation and injury. At least two animal studies (29) (30) have shown promising results: Vitamin E supplementation at supraphysiologic doses for a minimum of 5 weeks can decrease lipid peroxide levels with exhaustive exercise. To be effective at all, vitamin E must be given for at least 2 weeks before exercise, and five times the RDA for vitamin $\mathrm{E}$ may be necessary to prevent free-radical damage.(30).

\section{In Tabel 5:}

The exercise duration for the vitamin $\mathrm{E}$ groups subjects was significantly longer than the vitamin $\mathrm{C}$ groups subjects (Table5 ). Exercise duration after the vitamin supplementation treatment regimen was also significantly greater for the vitamin E group.

The major function of vitamin $\mathrm{E}$ is to work as a chainbreaking antioxidant in a fat soluble environment so as to protect polyunsaturated fatty acids within membrane phospholipids and in plasma lipoproteins. The purpose of this critical review was to determine whether vitamin $\mathrm{E}$ supplementation decreases exercise-induced lipid peroxidation in humans. If vitamin $\mathrm{E}$ alone is ineffective, researchers can turn their efforts to other individual antioxidants or combinations (29)

\section{Conclusions:}

Within the limits of the research sample and through the research results the following are shown:

1. The test workload (maximum physical effort) Cause in increased production of free radicals, based on the (Malondialdehyde)

2. Both of Vitamin (C) and (E) had a positive impact on MaloneAldehde, Lactic acid, VO2 max and Exercise duration in reducing the productivity of free radicals.

3. Vitamin ( E ) was more effective in increasing the performance during the test workload (maximum physical effort) 
4. Very important for athletes who are exposed repeatedly to high-intensity loads take a extra Antioxidants supplementation

\section{Recommendations:}

Through research result the research group recommends the following:

1. Attention to the seriousness of the high level of free radicals, as may be caused the risk of the lives of athletes

2. Monitoring the effect of exposure to high- intensity loads and delayed of injuries healing because it can be caused by the high level of free radicals and the damage caused to the tissue at the athletes.

3. Study the effect other antioxidant like selenium and coenzyme Q10, beta carotene with exposure maximum physical effort.

4. Study the effect of Combined of Antioxidant supplementation and functions of the circulatory system and respiratory during effort.

\section{References}

1. Beatty S, Koh HH, Phil M, Henson D, et al. The Role of oxidative stress in the pathogenesis of agerelated macular degeneration. Surv. Ophthalmol. 2000; 45: 115-134.

2. Bieri JG. Vitamin E. In: Brown ML, ed. Present knowledge in nutrition. Washington, DC: International Life Sciences Institute, 1990: 117-21.

3. Braakhuis, Andrea J. PhD, MND, APD, Effect of Vitamin C Supplements on Physical Performance American College of Sports Medicine, July/August 2012 - Volume 11 - Issue 4 - p 180-184

4. Child RB, Wilkinson DM, Fallowfield JL," Resting serum antioxidant status is positively correlated with peak oxygen uptake in endurance trained runners, $\mathrm{J}$ Sports Med Phys Fitness. 1999 Dec; 39(4):282-4.

5. D. Thompson, C. Williams, P. Garcia-Roves, S. J. McGregor, F.McArdle, M. J. Jackson "Post-exercise vitamin $\mathrm{C}$ supplementation and recovery from demanding exercise" European Journal of Applied Physiology , May 2003, pp 393 - 400

6. Davies KJ, Quintanilha AT, Brooks GA, et al: Free radicals and tissue damage produced by exercise. Biochem Biophys Res Commun 1982;107(4):11981205

7. Diplock AT: Dietary supplementation with antioxidants: is there a case for exceeding the recommended dietary allowances? Free Radic Biol Med 1987;3(3):199-201

8. Duthie GG, Robertson JD, Maughan RJ, et al: Blood antioxidant status and erythrocyte lipid peroxidation following distance running. Arch Biochem Biophys 1990;282(1):78-83

9. El-Kady ,M and AZIZ, A. The effect of physical exercise on DNA and some biochemical change for distance swimmer and runner, Congress International Medicine of sport, Paris - UNESCO , December 2000, Vol 15, no6:334.

10. Frei B, England L, Ames BN: Ascorbate is an outstanding antioxidant in human blood plasma. Proc Natl Acad Sci USA 1989;86(16):6377-6381

11. Gleeson M, Robertson JD, Maughan RJ. Influence of exercise on ascorbic acid status in man. Clin Sci 1987;73:501-5.

12. Gohil K, Packer L, de Lumen B, et al: Vitamin E deficiency and vitamin $\mathrm{C}$ supplements: exercise and mitochondrial oxidation. J Appl Physiol 1986;60(6):1986-1991

13. K. Birch, D. MacLaren, K. George, Sport and Exercise Physiology, Garland Science/BIOS Scientific Publishers, 2005,126-127

14. Kanter M: Free radicals and exercise: effects of nutritional antioxidant supplementation. Exerc Sport Sci Rev 1995;23:375-397

15. Kanter MM, Nolte LA, Holloszy JO: Effects of an antioxidant vitamin mixture on lipid peroxidation at rest and postexercise. J Appl Physiol 1993;74(2):965-969

16. Kwiterovich PO: The effect of dietary fat, antioxidants, and pro-oxidants on blood lipids, lipoproteins, and atherosclerosis. J Am Diet Assoc 1997;97(7 suppl):S31-S41

17. Linder MC (ed): Nutritional Biochemistry and Metabolism: With Clinical Applications, New York City, Elsevier, 1985, pp 176-178

18. Lovlin R, Cottle W, Pyke I, et al: Are indices of free radical damage related to exercise intensity? Eur J Appl Physiol Occup Physiol 1987;56(3):313-316

19. McBride JM, Kraemer WJ, Triplett-McBride T, et al: The effect of resistance exercise on free radical production. Med Sci Sports Exerc 1998;30(1):67-72

20. Meyers DG, Maloley PA, Weeks D: Safety of antioxidant vitamins. Arch Intern Med 1996;156(9):925-935

21. Odeh RM, Cornish LA: Natural antioxidants for the prevention of atherosclerosis. Pharmacotherapy 1995;15(5):648-659

22. Pyke S, Lew H, Quintanilha A: Severe depletion in liver glutathione during physical exercise. Biochem Biophys Res Commun 1986;139(3):926-931

23. Saxton JM, Donnelly AE, Roper HP: Indices of freeradical-mediated damage following maximum 
voluntary eccentric and concentric muscular work. Eur J Appl Physiol Occup Physiol 1994;68(3):189193

24. Sharon A. Plowman, Denise L. Smith, Exercise Physiology, FOR HEALTH, FITNESS, AND PERFORMANCE, Lippincott Williams \& Wilkins, 3ed, 2011.p139

25. Sumida S, Tanaka K, Kitao H, et al: Exerciseinduced lipid peroxidation and leakage of enzymes before and after vitamin $\mathrm{E}$ supplementation. Int $\mathbf{J}$ Biochem 1989;21(8):835-838

26. Ting HH, Timimi FK, Haley EA, et al: Vitamin C improves endothelium-dependent vasodilation in forearm resistance vessels of humans with hypercholesterolemia. Circulation 1997;95(12):2617-2622

27. Urso M.L., P.M.Clarkson (2003) Oxidative stress, exercise, and antioxidant supplementation. Toxicology 189:41-54

28. Victor L. Katch, William D. McArdle, Frank I Katch, Essentials of Exercise Physiology,4ed Lippincott W\&W,2011p 55-59.

29. Viitala P, Newhouse IJ. Vitamin E supplementation, exercise and lipid peroxidation in human participants,Eur J Appl Physiol. 2004 Oct;93(12):108-15. Epub 2004 Jul 10

30. Yu BP: Cellular defenses against damage from reactive oxygen species. Physiol Rev 1994;74(1):139-162 
